3 sextactic points are contacts of tangents from the flexes $P_{3}$. The 6 contacts of tangents from the sextactic points are the points $P_{12}$. The 12 contacts of tangents from $P_{12}$ in turn are the points $P_{24}$, and so on ad infinitum.

UNIVERSITY OF OREGON.

\title{
RELATED INVARIANTS OF TWO RATIONAL SEXTICS.
}

BY PROFESSOR J. E. ROWE.

(Read before the American Mathematical Society September 4, 1918.)

LET the parametric equations of the $R_{3}{ }^{6}$, the rational curve of order six in three dimensions, be

$$
\begin{array}{r}
x_{i}=\delta^{6}{ }_{i t} \equiv a_{\imath} t^{6}+6 b_{i} t^{5}+15 c_{\imath} t^{4}+20 d_{i} t^{3}+15 e_{i} t^{2}+ \\
6 f_{i} t+g_{i} \quad(i=1,2,3,4),
\end{array}
$$

and let the parametric equations of the $R_{2}{ }^{6}$, the rational plane curve of order six, be of the form

$$
\begin{aligned}
& x_{1}=\alpha_{t}^{6} \equiv a+b t+c t^{2}+d t^{3}+e t^{4}+f t^{5}+g t^{6}, \\
& x_{2}=\beta_{t}^{6} \equiv a^{\prime}+b^{\prime} t+c^{\prime} t^{2}+d^{\prime} t^{3}+e^{\prime} t^{4}+f^{\prime} t^{5}+g^{\prime} t^{6}, \\
& x_{3}=\gamma_{t}^{6} \equiv a^{\prime \prime}+b^{\prime \prime} t+c^{\prime \prime} t^{2}+d^{\prime \prime} t^{3}+e^{\prime \prime} t^{4}+f^{\prime \prime} t^{5}+g^{\prime \prime} t^{6} .
\end{aligned}
$$

It is well known that all plane sections of the $R_{3}{ }^{6}$ are apolar to a doubly infinite system of binary sextics, and that all line sections of the $R_{2}{ }^{6}$ are apolar to a triply infinite system of binary sextics. We shall let the four binary sextics $\delta_{i t}{ }^{6}$ of (1) be four linearly independent sextics of the apolar system of the $R_{2}{ }^{6}$, and the $\alpha_{t}{ }^{6}, \beta_{t}{ }^{6}, \gamma_{t}{ }^{6}$ of (2) be three linearly independent sextics of the apolar system of the $R_{3}{ }^{6}$. Our purpose is to point out briefly the relation between the invariants of the $R_{2}{ }^{6}$ and the invariants* of the $R_{3}{ }^{6}$.

By means of the twelve equations

* This relation must not be confused with the correspondence between invariants of the $R_{2}{ }^{n}$ and covariant surfaces of the $R_{3}{ }^{n}$. 


$$
\begin{array}{r}
a_{i} a-b_{i} b+c_{i} c-d_{i} d+e_{i} e-f_{i} f+g_{i} g=0 \\
a_{i} a^{\prime}-b_{i} b^{\prime}+c_{i} c^{\prime}-d_{\imath} d^{\prime}+e_{i} e^{\prime}-f_{i} f^{\prime}+g_{i} g^{\prime}=0, \\
a_{i} a^{\prime \prime}-b_{i} b^{\prime \prime}+c_{i} c^{\prime \prime}-d_{i} d^{\prime \prime}+e_{i} e^{\prime \prime}-f_{i} f^{\prime \prime}+g_{i} g^{\prime \prime}=0 \\
(i=1,2,3,4),
\end{array}
$$

it may be easily proved that the four-rowed determinants of the matrix of the coefficients of $\delta_{i t}{ }^{6}$ of the type $|a b c d|$ are proportional to the complementary three-rowed determinants of the matrix of the coefficients of $\alpha_{t}{ }^{6}, \beta_{t}{ }^{6}, \gamma_{t}{ }^{6}$ of the type $\left|e f^{\prime} g^{\prime \prime}\right|$. Let $T$ denote the substitution of the three-rowed determinants of (2) for the proportional four-rowed determinants of (1), and $T^{-1}$ the inverse substitution.

Invariants of the $R_{3}{ }^{6}$ are combinants of the four sextics $\delta_{i t}{ }^{6}$, and conversely, and these are rationally expressible in terms of the determinants of the type $|a b c d|$. Invariants of the $R_{2}{ }^{6}$ are combinants of $\alpha_{t}{ }^{6}, \beta_{t}{ }^{6}, \gamma_{t}{ }^{6}$, and conversely, and these are rationally expressible in terms of the determinants of the type $\left|a b^{\prime} c^{\prime \prime}\right|$. The combinants of $\delta_{i t}{ }^{6}$ are implicit invariants of the $R_{2}{ }^{6}$ which become explicit invariants of the $R_{2}{ }^{6}$ after the application of $T$. Similarly, combinants of $\alpha_{t}^{6}$, $\beta_{t}{ }^{6}, \gamma_{t}{ }^{6}$ are implicit invariants of the $R_{3}{ }^{6}$ which are transformed into explicit invariants of the $R_{3}{ }^{6}$ by means of $T^{-1}$. Hence any explicit invariant $I$ of the $R_{3}{ }^{6}$ is transformed into an explicit invariant $I^{\prime}$ of the $R_{2}{ }^{6}$ by means of $T$. Similarly, $T^{-1} I^{\prime}=I$. It is evident that the order of $I$ in the $|a b c d|$ is the same as that of $I^{\prime}$ in the $\left|a b^{\prime} c^{\prime \prime}\right|$. We shall now mention a few illustrations of this relation.

If $U^{\prime}$ is the undulation invariant of the $R_{2}^{6}, T^{-1} U^{\prime}=U$ is the stationary line invariant of the $R_{3}{ }^{6}$. From $P$, the pentatactic plane invariant of the $R_{3}{ }^{6}$, we obtain $T P=P^{\prime}$, the cusp invariant of the $R_{2}{ }^{6}$. Similarly, from $Q$, the quinquesecant line invariant of the $R_{3}{ }^{6}$, we derive $T Q=Q^{\prime}$ whose vanishing defines an $R_{2}{ }^{6}$ such that any six of its collinear points have parameters apolar to a binary quintic. If $N=0$ is the necessary and sufficient condition that the $R_{3}{ }^{6}$ have a node, $T N=N^{\prime}=0$ defines an $R_{2}{ }^{6}$ which has one secant that cuts out a cyclotomic set of parameters.

Pennsylvania State College, May, 1918. 